\title{
Partial Contact of a Rigid Multisinusoidal Wavy Surface with an Elastic Half-Plane
}

\author{
Ivan Y. Tsukanov \\ Ishlinsky Institute for Problems in Mechanics RAS, Prospekt Vernadskogo, 101-1, Moscow, 119526, Russia \\ Correspondence should be addressed to Ivan Y. Tsukanov; ivan.yu.tsukanov@gmail.com
}

Received 7 May 2018; Revised 24 July 2018; Accepted 4 October 2018; Published 18 October 2018

Academic Editor: Patrick De Baets

Copyright (C) 2018 Ivan Y. Tsukanov. This is an open access article distributed under the Creative Commons Attribution License, which permits unrestricted use, distribution, and reproduction in any medium, provided the original work is properly cited.

The interaction effects, arising at partial contact of rigid multisinusoidal wavy surface with an elastic half-plane, are considered in the assumption of continuous contact configuration. The analytical exact and asymptotic solutions for periodic and nonperiodic contact problems for wavy indenters are derived. Continuous contact configuration, appearing at small ratios of amplitude to wavelength for cosine harmonics, leads to continuous oscillatory contact pressure distribution and oscillatory relations between mean pressure and a contact length. Comparison of periodic and nonperiodic solutions shows that long-range elastic interaction between asperities does not depend on a number of cosine wavelengths.

\section{Introduction}

Real rough surfaces are three-dimensional and multiscale. Besides fully random rough surfaces [1] there are natural and technical surfaces, having quasiregular character of asperities on several scales (e.g., periodic anisotropic waviness) [2]. For these surfaces the geometric model of two-dimensional wavy $2 \mathrm{D}$ profile can be applied as a first approximation. Also, in some fields of engineering, the wavy textures of different shapes are used (e.g., in optical devices and MEMS) [3]. Considering the elastic contact processes, occurring for soft materials (polymers and biological materials), the various analytical methods of plane elasticity can be applied. In the case of full contact, when the gap between surfaces is filled, the problem can be easily solved by Fourier transform method [4]. However, the very high applied pressure is required to reach the full contact condition even for soft materials, so partial contact is the more often case. Partial contact between wavy surfaces is a problem with mixed boundary conditions, which was solved by different mathematical techniques.

The classic periodic contact problem in plane elasticity is an old problem $[5,6]$. Concerning geometry of a wavy surface, considered in the previous studies, the cosine [7, 8], the squared cosine [9], and evenly spaced parabolic or wedge stamps $[10,11]$ were generally used. The analytical solution of the pointed problem for those surface geometries was obtained by different methods. For the cosine profile they are complex stress function [7], dual series equation [12], intercontact gaps method [13], variable transform method [11], and fracture mechanics approach [14].

Taking into account simple wavy geometry (cosine or squared cosine) the contact problems with more complicated boundary conditions were studied: sliding problem with friction $[15,16]$, with a fluid lubricant [17], with a partial slip $[11,18]$, with adhesion and sliding friction [19], for viscoelastic material [20], for Winkler model of viscoelastic material and adhesion [21], for elastic layer with presence of friction and wear [22], and dynamic problem for anisotropic half-plane [23].

The normal elastic problem for a two-dimensional nonsinusoidal wavy profile, where a shape of a waveform is controlled by a parameter, was solved analytically [24]. It was established that the pressure distribution is highly sensitive to the shape of a wavy surface, especially at large loads.

The presence of several scales of a wavy surface leads in general to a multizone periodic contact problem $[9,25]$. The asymptotic approximate solution for initial contact for a twoscale wavy surface was obtained [24]. It was shown that even 


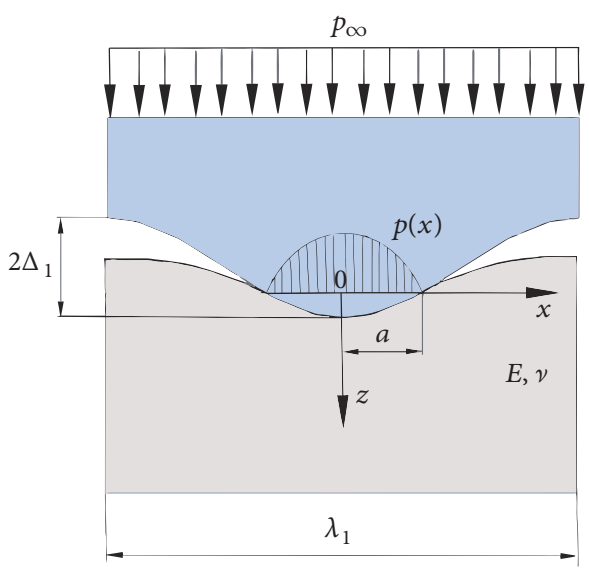

(a)

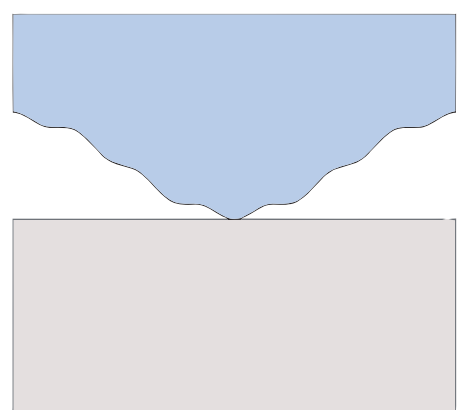

(b)

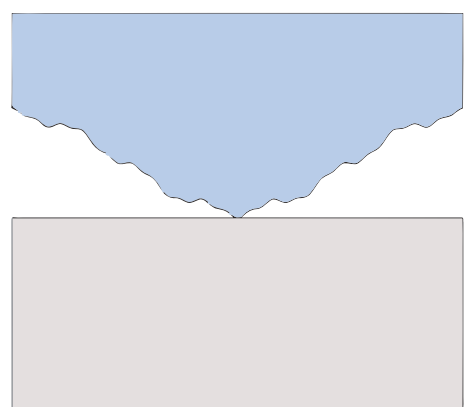

(c)

FIgure 1: Contact of a cosine wavy profile, having one (a), two (b), and three (c) harmonics with an elastic half-plane.

for initial contact the interplay between harmonics exists. Considering the problem in a wide range of applied loads it is necessary to use a numerical procedure. Such studies were performed by different techniques: Fourier series and cotangent transform [26], full contact solution and iteration procedure [27], FFT and variational principle [28], nonlinear boundary integral equation [29], boundary element method [30], and finite element method [31]. The equations for internal stresses for sinusoidal pressure distributions in $2 \mathrm{D}$ and $3 \mathrm{D}$ cases were also derived [32]. The results of these studies show that multiscale character of a wavy surface at partial contact with an elastic half-plane leads to multiple peaks of high pressure. The pressure distribution is jagged in this case, and a load-area dependence tends to proportionality at large number of harmonics [31].

In cases, considered in the previous studies, the contact was partial at all scales, because the amplitudes of different harmonics were comparable. This situation leads to a discontinuous (discrete) contact configuration [33]. Besides numerical methods, the other way to solve these problems is usage of multiasperity contact models. Based on the nature of the surfaces models can be deterministic and statistical. Review of statistical models, based on individual asperity contact, in comparison with the Persson's model and numerical simulations, is performed in [34]. For the nearly complete contact case, when the ratio of the real area of contact to the nominal contact area approaches unity, the statistical model, based on fracture mechanics approach, was developed [35]. For deterministic multiscale surfaces, (e.g., multisinusoidal self-affine surfaces), the Archard's approach was successfully implemented [36, 37].

However, if at a certain scale amplitude of the cosine harmonic is much smaller than its period, full contact on this scale occurs, and a continuous oscillating pressure distribution on a larger scale will be observed [33, 38]. For distinguishing these cases the Johnson parameter, coupling an amplitude, a period of cosine harmonic, and a reduced modulus of elasticity with Hertzian pressure at the point, where maximum pressure occurs, is used [33]. In the present study the continuous contact configuration, observed at small amplitudes of subsequent cosine harmonics, is analyzed analytically for periodic and nonperiodic multisinusoidal rigid indenters in contact with an elastic half-plane.

\section{Problem Formulation and Assumptions}

The general scheme of the problem on the single period $\lambda_{1}$ for one, two, and three cosine harmonics profile is presented in Figure 1.

The wavy surface is assumed to be rigid, and the elastic half-plane is an isotropic semi-infinite body with two elastic constants: Young's modulus $E$ and Poisson's coefficient $\nu$. Also the plain strain condition is applied. The amplitudes of cosine harmonics are much smaller than their periods $\left(\Delta_{i} \ll\right.$ $\lambda_{i}$, where $i=1,2 \ldots N$ is a harmonic sequence number). This condition makes it possible to apply the linear elasticity theory. The Johnson parameter $\chi=\pi E \Delta_{i} / 2 p_{h} \lambda_{i}$ (where $p_{h}=$ $2 p_{\infty} \lambda_{i} / \pi a, p_{\infty}$ is an applied mean pressure, and $a$ is a contact half-width) should be $\chi<1$ [33] for preserving the continuous contact configuration.

The two different problems with similar geometry of a rigid surface are considered. For the problem with periodic boundary conditions the integral equation with Hilbert kernel is used [11]:

$$
\frac{E}{2\left(1-v^{2}\right)} \frac{\partial h(x)}{\partial x}=\frac{1}{2 \pi} \int_{-a}^{a} p(\xi) \cot \frac{x-\xi}{2} d \xi,
$$

where $h(x)$ is an initial gap between surfaces, and $p(x)$ is a contact pressure distribution.

For a nonperiodic indenter the integral equation with Cauchy kernel is used [10]:

$$
\frac{E}{2\left(1-v^{2}\right)} \frac{\partial h(x)}{\partial x}=\frac{1}{2 \pi} \int_{-a}^{a} \frac{p(\xi)}{x-\xi} d \xi .
$$

Choosing for simplicity the largest wavelength $\lambda_{1}=2 \pi$, one can write the expression for the gap function derivative for the $i$ th cosine harmonic:

$$
\frac{\partial h_{i}(x)}{\partial x}=\frac{\partial}{\partial x}\left(\delta-\Delta_{i}\left(1-\cos n_{i} x\right)\right)=-\Delta_{i} n_{i} \sin n_{i} x,
$$


where $\delta$ is a contact approach and $n_{i}=\lambda_{1} / \lambda_{i}$. In the given formulation of the problem $n_{i} \in \mathbb{N}$.

So, on the basis of the superposition principle and taking into account the assumed continuous contact configuration the total contact pressure distribution can be obtained as a sum of distributions of separate cosine harmonics:

$$
p(x)=\frac{E}{2\left(1-v^{2}\right)} \sum_{i=1}^{N} p_{i}(x),
$$

where $N$ is a number of wavelengths and $p_{i}(x)$ is a component of pressure distribution for the $i$ th wavelength.

The vertical elastic displacements can be obtained via the following expression $[9,10]$ :

$$
\begin{aligned}
\bar{u}_{z}(x) & =\frac{2\left(1-v^{2}\right)}{\pi E} \int_{-\infty}^{\infty} p(\xi) \ln |x-\xi| \mathrm{d} \xi+C \\
& =\frac{2\left(1-v^{2}\right)}{\pi E} \sum_{i=1}^{N} \bar{u}_{z i}(x)+C,
\end{aligned}
$$

where $C$ is a constant depending on the selected datum point and $\bar{u}_{z i}(x)$ are the surface vertical displacements for the $i$ th wavelength.

The mean (nominal) pressure $p_{\infty}$ is determined by invoking the equilibrium equation:

$$
p_{\infty}=\frac{E}{\left(1-v^{2}\right)} \sum_{i=1}^{N} p_{\infty i}=\frac{1}{4 \pi} \frac{E}{\left(1-v^{2}\right)} \sum_{i=1}^{N} \int_{-a}^{a} p_{i}(x) d x,
$$

where $p_{\infty i}$ is a component of mean pressure corresponding to the $i$ th wavelength.

\section{Solutions of the Problem for the $i$ th Harmonic}

3.1. Solution for a Periodic Wavy Surface. Following equations (1) and (3) the integral equation for the $i$ th harmonic is as follows:

$$
-\Delta_{i} n_{i} \sin n_{i} x=\frac{1}{\pi} \int_{-a}^{a} p_{i}(\xi) \cot \frac{x-\xi}{2} d \xi .
$$

The analytical solution for the contact pressure distribution for the $i$ th harmonic can be obtained via the reduction of equation (7) to the integral equation with Cauchy kernel using the following variable transform [11]:

$$
\begin{aligned}
& u=\tan \frac{\xi}{2} ; \\
& v=\tan \frac{x}{2} ; \\
& \alpha=\tan \frac{a}{2} .
\end{aligned}
$$

Considering the symmetry of the profile the integral equation (7) is reduced to

$$
-\Delta_{i} n_{i} \frac{2 v}{1+v^{2}} U_{n_{i}-1}\left(\frac{1-v^{2}}{1+v^{2}}\right)=\frac{1}{\pi} \int_{-\alpha}^{\alpha} \frac{p_{i}(u)}{v-u} d u,
$$

where $U_{n_{i}}$ - is a Chebyshev polynomial of the second kind with a degree $n_{i}$ [39].

Taking into account the considered assumptions the solution of equation (9) can be obtained by means of the Chebyshev expansion of the left side and the known spectral relations for the Chebyshev polynomials (Appendix). In initial variables the contact pressure distribution for the $i$ th harmonic is determined by

$$
\begin{aligned}
& p_{i}(x) \\
& =\Delta_{i} n_{i} \sqrt{1-\left(\frac{\tan (x / 2)}{\tan (a / 2)}\right)^{2}} \sum_{j=1}^{\infty} A_{i j} U_{j-1}\left(\frac{\tan (x / 2)}{\tan (a / 2)}\right),
\end{aligned}
$$

where

$$
\begin{aligned}
A_{i j} & =\frac{2}{\pi} \int_{-1}^{1} \frac{\varphi_{i}(s) T_{j}(s)}{\sqrt{1-s^{2}}} d s, \quad j=1,2, \ldots ; \\
\varphi_{i}(s) & =\frac{\tan (a / 2) s}{1+(\tan (a / 2) s)^{2}} U_{n_{i}-1}\left(\frac{1-(\tan (a / 2) s)^{2}}{1+(\tan (a / 2) s)^{2}}\right),
\end{aligned}
$$

where $T_{j}$ - is a Chebyshev polynomial of the first kind with a degree $j$ [39].

The total pressure distribution is obtained by using equation (4). For numerical calculations it is necessary to hold finite terms of the infinite series in equation (10). For the arbitrary period, the variables $x$ and $a$ in equations (10) and (12) should be multiplied on $2 \pi / \lambda_{1}$. The mean pressure $p_{\infty i}$ and the vertical displacements $\bar{u}_{z i}(x)$ can be obtained using numerical integration in equations (5) and (6). The maximum pressure is determined as the pressure at the point $x=0$.

3.2. Solution for a Wavy Rigid Nonperiodic Indenter. Following equations (2) and (3) the integral equation for the $i$ th harmonic is

$$
-\Delta_{i} n_{i} \sin n_{i} x=\frac{1}{\pi} \int_{-a}^{a} \frac{p_{i}(\xi)}{x-\xi} d \xi
$$

The solution of the equation (13) can be obtained, using an inversion without singularities on both endpoints $[8,11]$ and the Chebyshev expansion of the left side [39], which can be written explicitly:

$$
\begin{aligned}
& p_{i}(x) \\
& \quad=\Delta_{i} n_{i} \sqrt{1-\left(\frac{x}{a}\right)^{2}} \sum_{j=0}^{\infty}(-1)^{-j} \mathrm{~J}_{2 j+1}\left(a n_{i}\right) U_{2 j}\left(\frac{x}{a}\right),
\end{aligned}
$$

where $\mathrm{J}_{j}(t)$ is the Bessel function of the first kind of the integer order $j$ and the argument $t$ [39].

The displacements within the contact zone $x \in[-a, a]$ can be determined analytically using equation (5) and the 
relations for Chebyshev polynomials [40]. For the ith harmonic the final relation is

$$
\begin{gathered}
\bar{u}_{z i}(x)=-\Delta_{i} n_{i} a\left[\mathrm{~J}_{1}\left(a n_{i}\right)\left(\frac{x^{2}}{a^{2}}-\frac{1}{2}-\ln \frac{2}{a}\right)\right. \\
+\sum_{j=3}^{\infty} /(-1)^{(1-j) / 2} \mathrm{~J}_{j}\left(a n_{i}\right) \\
\left.\cdot\left(\frac{T_{j+1}(x / a)}{j+1}-\frac{T_{j-1}(x / a)}{j-1}\right)\right],
\end{gathered}
$$

where $\operatorname{sign} \sum^{/}$identifies the sum of terms with odd $j$ only.

According to equation (6) the mean pressure for the $i$ th harmonic $p_{\infty i}$ is calculated by integration of equation (14) and resulting in a simple expression:

$$
p_{\infty i}=0.25 \Delta_{i} a n_{i} \mathrm{~J}_{1}\left(a n_{i}\right)
$$

The approximate close-form relation for the contact pressure distribution can be obtained assuming that the largest values of pressure are concentrated near the point $x$ $=0$. Then equation (14) can be represented as

$$
\begin{aligned}
p_{i}(x)= & \Delta_{i} n_{i} \sqrt{1-\left(\frac{x}{a}\right)^{2}} \frac{\partial}{\partial x} \\
& \cdot \sum_{j=0}^{\infty} /(-1)^{(1-j) / 2} a \mathrm{~J}_{j}\left(a n_{i}\right) \frac{T_{j}(\sin (x / a))}{j},
\end{aligned}
$$

where $\operatorname{sign} \sum^{\prime}$ identifies the sum of terms with odd $j$ only. Using the known relations for the Chebyshev polynomials [41] the following expression can be written:

$$
\begin{aligned}
& p_{i}(x) \\
& =\Delta_{i} n_{i} \sqrt{1-\left(\frac{x}{a}\right)^{2}} \sum_{j=0}^{\infty} \mathrm{J}_{2 j+1}\left(a n_{i}\right) \cos \left((2 j+1)\left(\frac{x}{a}\right)\right) .
\end{aligned}
$$

With the use of an approximate relation between zeros of Bessel functions of integer order [42] the following expression can be written:

$$
\begin{aligned}
p_{i}(x) & \approx \Delta_{i} n_{i} \sqrt{1-\left(\frac{x}{a}\right)^{2}}\left[\mathrm{~J}_{1}\left(a n_{i}\right)\right. \\
& \left.+\sum_{j=2}^{\infty} \mathrm{J}_{2 j}\left(a n_{i}+1\right) \cos \left(2 j\left(\frac{x}{a}\right)\right)\right] .
\end{aligned}
$$

Then, applying the Jacobi-Anger expansion [42], the close-form relation is

$$
\begin{aligned}
& p_{i}(x) \approx \Delta_{i} n_{i} \sqrt{1-\left(\frac{x}{a}\right)^{2}}\left[\mathrm{~J}_{1}\left(a n_{i}\right)\right. \\
& +\mathrm{J}_{2}\left(a n_{i}+1\right) \cos \left(2\left(\frac{x}{a}\right)\right) \\
& \left.+0.5 \cos \left(\left(a n_{i}+1\right) \sin \left(\frac{x}{a}\right)\right)-\mathrm{J}_{0}\left(a n_{i}+1\right)\right] .
\end{aligned}
$$

The close-form integral relation for a maximum pressure $(x=0)$ can be determined exactly from equation (18):

$$
p_{\text {imax }}=\Delta_{i} n_{i} \sum_{j=0}^{\infty} \mathrm{J}_{2 j+1}\left(a n_{i}\right)=0.5 \Delta_{i} n_{i} \int_{0}^{a n_{i}} \mathrm{~J}_{0}(t) d t .
$$

\section{Results and Discussion}

The evolution of the dimensionless contact pressure distribution $p(x) / p *\left(p *=\pi \mathrm{E} \Delta_{1} / \lambda_{1}\right)$ for a periodic problem (equations ((6) and (10)-(12)), $\lambda_{1}=2 \pi, \Delta_{1}=0.5$ ) for various contact lengths $(2 a)$ and two different profiles $f(x)$ is shown in Figure 2.

The exact (solid lines, equation (14)) and the approximate (dotted lines, equation (20)) graphs of the dimensionless contact pressure $p(x) / p *$ for different profiles of a nonperiodic wavy indenter are shown in Figure 3.

Figures 2 and 3 illustrate that, with increasing the number of harmonics, the pressure distribution becomes more complex and the maximum pressure grows significantly. For a single-scale periodic cosine profile (Figure 2(a)) the Westergaard's solution is recovered. For a single-scale nonperiodic indenter (Figure 3(a)) the Hertz solution is observed, as the cosine function is very close to the quadratic parabola. Thereby, the distributions, presented in Figures 3(b) and $3(\mathrm{c})$, correspond to wavy cylinder problem at small waviness [38]. Comparison of the exact and the approximate values of pressure for a single indenter (Figure 3 ) shows that equation (20) satisfactorily describes the behavior of the pressure distribution.

Comparing the periodic and the nonperiodic solutions the elastic interaction effect is of interest. The mean pressure - contact length curves for two profiles, calculated from periodic and nonperiodic solutions, are presented in Figure 4.

Figure 4 shows that, at small contact lengths $(2 a<$ $\left.0.25 \lambda_{1}\right)$, the solutions are close. The piece of graphs agreement does not depend on profile geometry. With increase of load the periodic solution gives the smaller contact length due to elastic interaction on the largest scale. For the profile with two cosine harmonics and continuous contact configuration, presented in this study, the oscillations of mean pressure contact length curves are observed (Figure 4(b)). Curves in Figure 4(a) correspond to Westergaard's (curve 1) and Hertz (curve 2) solutions, recovered for profile with one wavelength.

Graphs of the mean and the maximum pressures versus contact length on the interval $2 a<0.25 \lambda_{1}$ for different profiles of a wavy nonperiodic indenter are shown in Figure 5. 


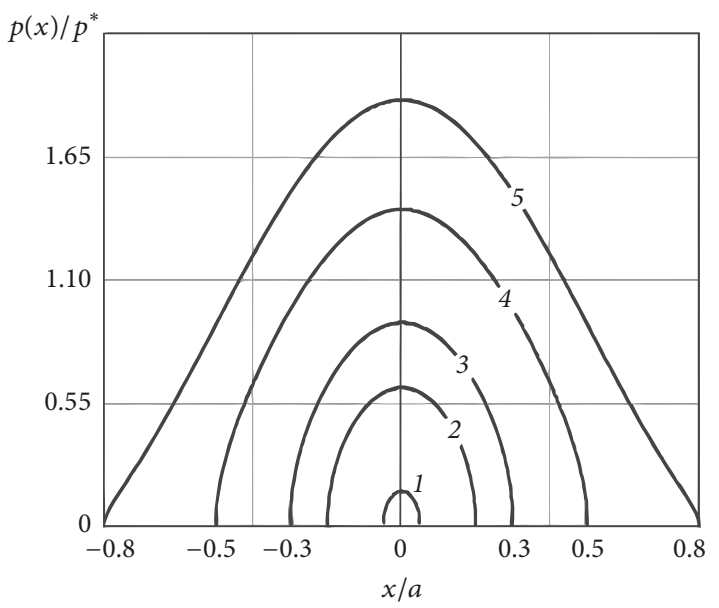

(a)

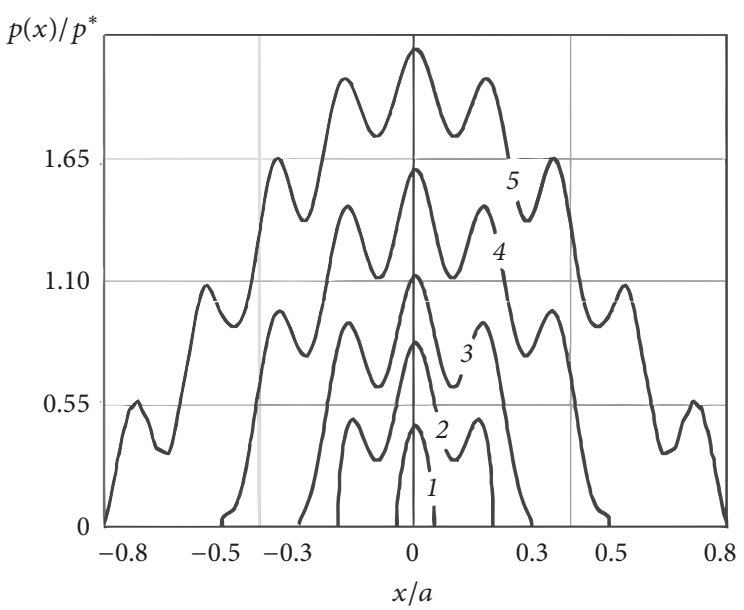

(b)

FIGURE 2: Evolution of contact pressure distribution for wavy periodic profile $\left(\lambda_{1}=2 \pi, \Delta_{1}=0.5\right): f(x)=\Delta_{1} \cos (x)(\mathrm{a}) ; f(x)=$ $\Delta_{1} \cos (x)+0.02 \Delta_{1} \cos (11 x)(\mathrm{b}) ; 2 a=0.05 \lambda_{1}(1) ; 2 a=0.2 \lambda_{1}(2) ; 2 a=0.3 \lambda_{1}(3) ; 2 a=0.5 \lambda_{1}(4) ; 2 a=0.8 \lambda_{1}(5)$.

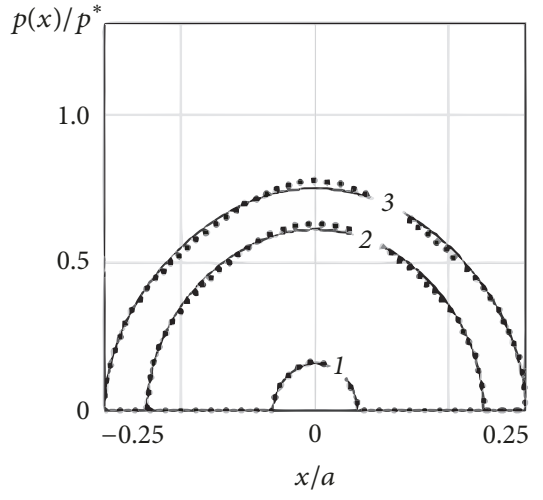

(a)

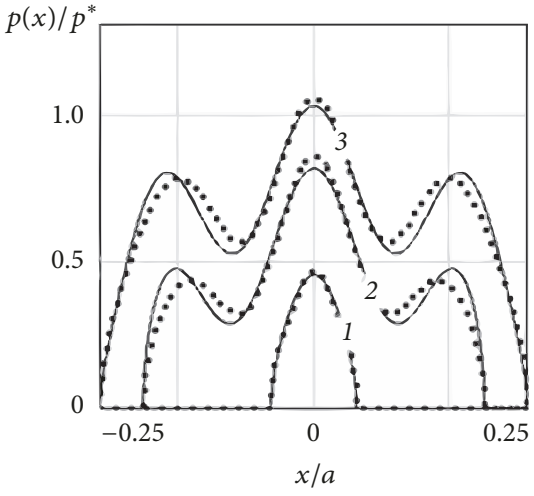

(b)

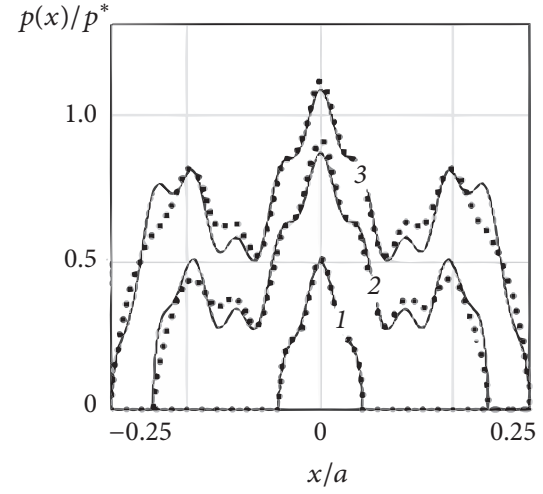

(c)

FIGURE 3: Evolution of contact pressure distribution for a nonperiodic wavy indenter $\left(\lambda_{1}=2 \pi, \Delta_{1}=0.5\right): f(x)=\Delta_{1} \cos (x)(\mathrm{a}) ; f(x)=$ $\Delta_{1} \cos (x)+0.02 \Delta_{1} \cos (11 x)(\mathrm{b}) ; f(x)=\Delta_{1} \cos (x)+0.02 \Delta_{1} \cos (11 x)+0.0015 \Delta_{1} \cos (40 x)$ (c); $2 a=0.05 \lambda_{1}(1) ; 2 a=0.2 \lambda_{1}(2) ; 2 a=0.25 \lambda_{1}(3)$.

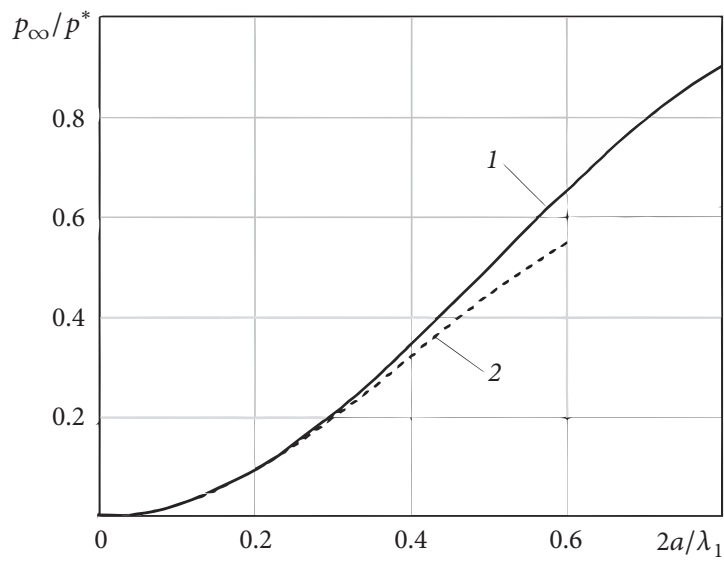

(a)

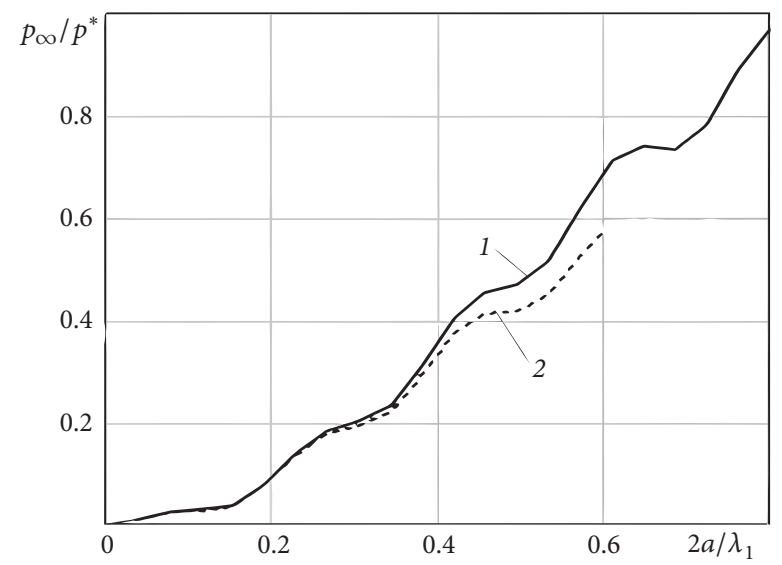

(b)

FIgURE 4: Graphs of dimensionless mean pressure as a function of dimensionless contact length $\left(\lambda_{1}=2 \pi, \Delta_{1}=0.5\right): f(x)=\Delta_{1} \cos (x)(\mathrm{a})$; $f(x)=\Delta_{1} \cos (x)+0.02 \Delta_{1} \cos (11 x)(b) ; 1-$ periodic solution, 2 - nonperiodic solution. 


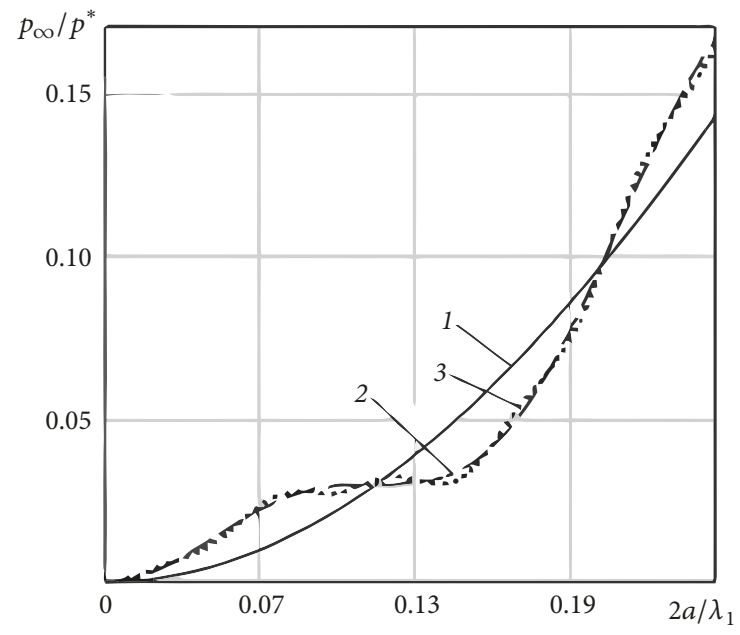

(a)

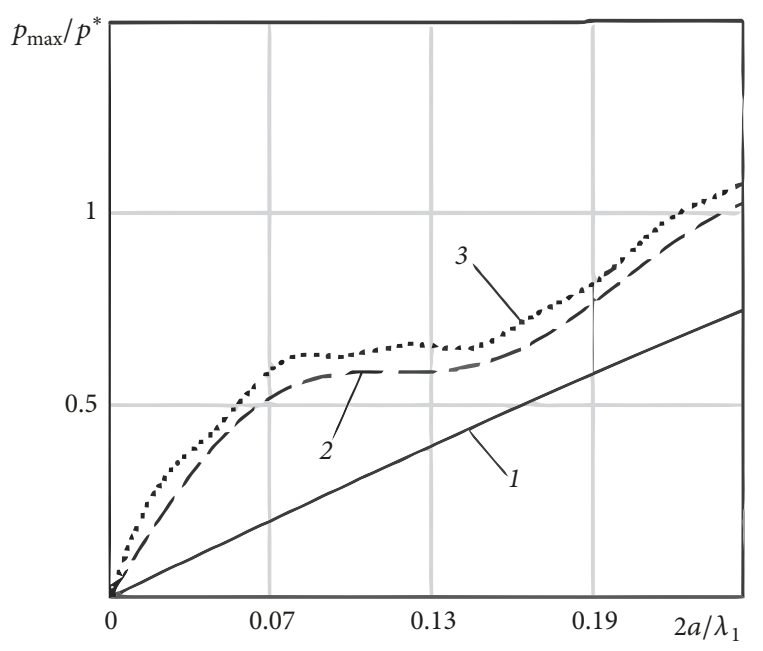

(b)

FIGURE 5: Dimensionless mean pressure (a) and maximum pressure (b) as a function of dimensionless contact length for profiles with different numbers of cosine harmonics $\left(\lambda_{1}=2 \pi, \Delta_{1}=0.5\right)$ : $f(x)=\Delta_{1} \cos (x)(1) ; f(x)=\Delta_{1} \cos (x)+0.02 \Delta_{1} \cos (11 x)(2) ; f(x)=$ $\Delta_{1} \cos (x)+0.02 \Delta_{1} \cos (11 x)+0.0015 \Delta_{1} \cos (40 x)(3)$.

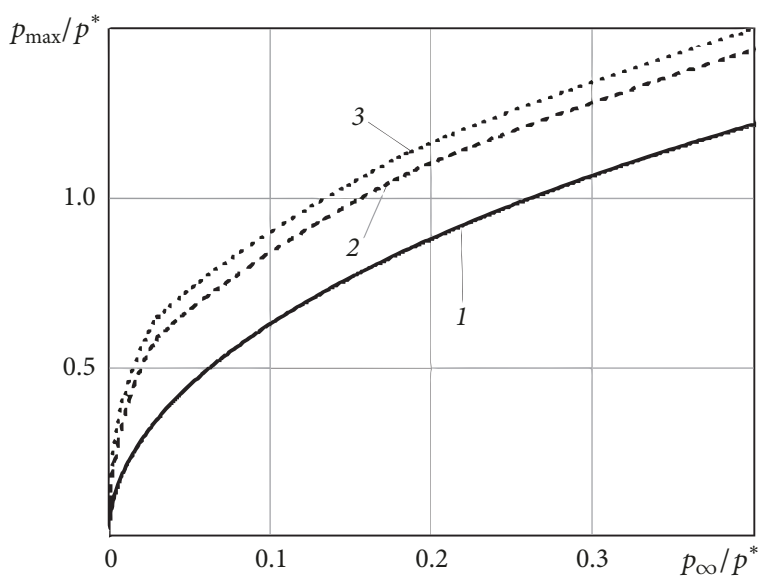

FIGURE 6: Dimensionless maximum pressure as a function of dimensionless mean pressure for different profiles $\left(\lambda_{1}=2 \pi, \Delta_{1}=\right.$ $0.5): f(x)=\Delta_{1} \cos (x)(1) ; f(x)=\Delta_{1} \cos (x)+0.02 \Delta_{1} \cos (11 x)(2) ; f(x)$ $=\Delta_{1} \cos (x)+0.02 \Delta_{1} \cos (11 x)+0.0015 \Delta_{1} \cos (40 x)(3)$.

Figure 5 shows that the maximum pressure depends on profile geometry stronger than the mean pressure. However, adding the third harmonic leads to insignificant change of the graphs character. Continuous contact configuration at the presence of several cosine wavelengths leads to oscillatory character of the mean and the maximum pressure graphs. Combining these two graphs numerically one can obtain the dependence of peak pressure from mean pressure (Figure 6).

Figure 6 shows that dependences of the maximum pressure from the mean pressure are not oscillatory for the profiles with two and three wavelengths, and additional cosine harmonics change the graph considerably in value but not in character. This statement can be useful in the analysis of contact surfaces fracture processes [33].

\section{Conclusions}

The continuous contact configuration is one of the two possible configurations, arising at indentation of a multisinusoidal 2D wavy surface into an elastic half-plane. This configuration leads to continuous oscillatory contact pressure distribution. Comparison of the derived periodic and nonperiodic solutions shows that the long-range elastic interaction between asperities does not depend on a number of cosine wavelengths and can be neglected at small loads (contact lengths) for arbitrary wavy profile geometry. The assumption of neglecting the long-range periodicity leads to exact equations for determining the remote and the maximum pressures from the contact length, described by oscillatory functions. However, the dependences of the maximum pressure from the mean pressure are not oscillatory for the profiles with two and three wavelengths and resemble those for a simple cosine profile of indenter. The influence of the additional cosine harmonics on the maximum pressure is significantly larger than on the mean pressure for the same contact zone length. The derived equations can be used at the analysis of contact characteristics of deterministic profiles of arbitrary geometry and also at the validation of more complex numerical models of rough surfaces contact.

\section{Appendix}

\section{Derivation of Contact Pressure Distribution for the Periodic Problem}

The main integral equation of the considered contact problem for the $i$ th harmonic in transformed variables (8) is

$$
-\Delta_{i} n_{i} \frac{2 v}{1+v^{2}} U_{n_{i}-1}\left(\frac{1-v^{2}}{1+v^{2}}\right)=\frac{1}{\pi} \int_{-\alpha}^{\alpha} \frac{p_{i}(u)}{v-u} d u,
$$


where $U_{n}$ is a Chebyshev polynomial of a second kind with a degree $n_{i}$.

The appropriate inversion of this integral equation has to be nonsingular on both endpoints [11]:

$$
\begin{gathered}
p_{i}(v)=-\frac{\Delta_{i} n_{i}}{\pi} \sqrt{\alpha^{2}-v^{2}} \int_{-\alpha}^{\alpha} \frac{v}{1+v^{2}} U_{n_{i}-1}\left(\frac{1-v^{2}}{1+v^{2}}\right) \\
\cdot \frac{1}{\sqrt{\alpha^{2}-u^{2}}} \frac{1}{u-v} d u .
\end{gathered}
$$

By introducing the new variables,

$$
\begin{aligned}
& r=\frac{v}{\alpha}, \\
& s=\frac{u}{\alpha},
\end{aligned}
$$

the expression (A.2) can be written in the following form:

$$
p_{i}(r)=-\frac{\Delta_{i} n_{i}}{\pi} \sqrt{1-r^{2}} \int_{-1}^{1} \varphi_{i}(s) \frac{1}{\sqrt{1-s^{2}}} \frac{1}{s-r} d s,
$$

where the function $\varphi_{i}(s)$ is

$$
\varphi_{i}(s)=\frac{\alpha s}{1+\alpha^{2} s^{2}} U_{n_{i}-1}\left(\frac{1-\alpha^{2} s^{2}}{1+\alpha^{2} s^{2}}\right) .
$$

Since the integrand function is defined on the interval [-1; 1] and satisfies the Hölder condition, it can be represented as an expansion in Chebyshev polynomials of the first kind [41].

$$
\varphi_{i}(s)=\frac{A_{i 0}}{2}+A_{i 1} T_{1}(s)+A_{i 2} T_{2}(s)+\ldots,
$$

where $T_{j}$ is a Chebyshev polynomial of the first kind with a degree $j$ [39].

The coefficients $A_{i j}$ in equation (A.6) are defined by the following expression [43]:

$$
\begin{aligned}
& A_{i 0}=0 \\
& A_{i j}=\frac{2}{\pi} \int_{-1}^{1} \frac{\varphi_{i}(s) T_{j}(s)}{\sqrt{1-s^{2}}} d s, \quad j=1,2, \ldots ;
\end{aligned}
$$

With the use of integral relation between the Chebyshev polynomials of the first and the second kind [41]

$$
\frac{1}{\pi} \int_{-1}^{1} \frac{T_{j}(s)}{\sqrt{1-s^{2}}} \frac{1}{s-r} d s=U_{j-1}(r), \quad j=0,1,2, \ldots
$$

and equation (A.4) the expression for the contact pressure distribution for the $i$ th harmonic is

$$
p_{i}(r)=-\Delta_{i} n_{i} \sqrt{1-r^{2}} \sum_{j=1}^{\infty} A_{i j} U_{j-1}(r) .
$$

Returning to the original variables, and bearing in mind positive pressures notation, one can obtain

$$
\begin{aligned}
& p_{i}(x) \\
& =\Delta_{i} n_{i} \sqrt{1-\left(\frac{\tan (x / 2)}{\tan (a / 2)}\right)^{2}} \sum_{j=1}^{\infty} A_{i j} U_{j-1}\left(\frac{\tan (x / 2)}{\tan (a / 2)}\right) .
\end{aligned}
$$

\section{Data Availability}

No data were used to support this study.

\section{Conflicts of Interest}

The author declares that there are no conflicts of interest regarding the publication of this paper.

\section{Acknowledgments}

The research was supported by RSF (project no. 14-29-00198).

\section{References}

[1] B. N. J. Persson, "Theory of rubber friction and contact mechanics," The Journal of Chemical Physics, vol. 115, no. 8, pp. 3840-3861, 2001.

[2] A. C. Rodríguez Urribarrí, E. van der Heide, X. Zeng, and M. B. de Rooij, "Modelling the static contact between a fingertip and a rigid wavy surface," Tribology International, vol. 102, pp. 114-124, 2016.

[3] A. S. Adnan, V. Ramalingam, J. H. Ko, and S. Subbiah, "Nano texture generation in single point diamond turning using backside patterned workpiece," Manufacturing Letters, vol. 2, no. 1, pp. 44-48, 2013.

[4] Y. Ju and T. N. Farris, "Spectral Analysis of Two-Dimensional Contact Problems," Journal of Tribology, vol. 118, no. 2, p. 320, 1996.

[5] M. A. Sadowski, "Zwiedimensionale probleme der elastizitatshtheorie," ZAMM-Zeitschrift für Angewandte Mathematik und Mechanik, vol. 8, no. 2, pp. 107-121, 1928.

[6] N. I. Muskhelishvili, Some Basic Problems of the Mathematical Theory of Elasticity, Springer, Dordrecht, Netherlands, 1977.

[7] H. M. Westergaard, "Bearing pressures and cracks," Journal of Applied Mechanics, vol. 6, pp. 49-53, 1939.

[8] K. L. Johnson, Contact Mechanics, Cambridge University Press, Cambridge, UK, 1987.

[9] I. Y. Schtaierman, Contact Problem of Theory of Elasticity, Gostekhizdat, Moscow, Russia, 1949.

[10] L. A. Galin, Contact problems, Springer, Netherlands, Dordrecht, 2008

[11] J. M. Block and L. M. Keer, "Periodic contact problems in plane elasticity," Journal of Mechanics of Materials and Structures, vol. 3, no. 7, pp. 1207-1237, 2008.

[12] J. Dundurs, K. C. Tsai, and L. M. Keer, "Contact between elastic bodies with wavy surfaces," Journal of Elasticity, vol. 3, no. 2, pp. 109-115, 1973.

[13] A. A. Krishtafovich, R. M. Martynyak, and R. N. Shvets, "Contact between anisotropic half-plane and rigid body with regular microrelief," Journal of Friction and Wear, vol. 15, pp. 15-21, 1994.

[14] Y. Xu and R. L. Jackson, "Periodic Contact Problems in Plane Elasticity: The Fracture Mechanics Approach," Journal of Tribology, vol. 140, no. 1, p. 011404, 2018.

[15] E. A. Kuznetsov, "Periodic contact problem for half-plane allowing for forces of friction," Soviet Applied Mechanics, vol. 12, no. 10, pp. 1014-1019, 1976.

[16] M. Nosonovsky and G. G. Adams, "Steady-state frictional sliding of two elastic bodies with a wavy contact interface," Journal of Tribology, vol. 122, no. 3, pp. 490-495, 2000. 
[17] Y. A. Kuznetsov, "Effect of fluid lubricant on the contact characteristics of rough elastic bodies in compression," Wear, vol. 102, no. 3, pp. 177-194, 1985.

[18] M. Ciavarella, "The generalized Cattaneo partial slip plane contact problem. II. Examples," International Journal of Solids and Structures, vol. 35, no. 18, pp. 2363-2378, 1998.

[19] G. Carbone and L. Mangialardi, "Adhesion and friction of an elastic half-space in contact with a slightly wavy rigid surface," Journal of the Mechanics and Physics of Solids, vol. 52, no. 6, pp. 1267-1287, 2004.

[20] N. Menga, C. Putignano, G. Carbone, and G. P. Demelio, “The sliding contact of a rigid wavy surface with a viscoelastic halfspace," Proceedings of the Royal Society A Mathematical, Physical and Engineering Sciences, vol. 470, no. 2169, pp. 2014039220140392, 2014.

[21] I. G. Goryacheva and Y. Y. Makhovskaya, "Modeling of friction at different scale levels," Mechanics of Solids, vol. 45, no. 3, pp. 390-398, 2010.

[22] I. A. Soldatenkov, "The contact problem for an elastic strip and a wavy punch under friction and wear," Journal of Applied Mathematics and Mechanics, vol. 75, no. 1, pp. 85-92, 2011.

[23] Y.-T. Zhou and T.-W. Kim, "Analytical solution of the dynamic contact problem of anisotropic materials indented with a rigid wavy surface," Meccanica, vol. 52, no. 1-2, pp. 7-19, 2017.

[24] I. Y. Tsukanov, "Effects of shape and scale in mechanics of elastic interaction of regular wavy surfaces," Proceedings of the Institution of Mechanical Engineers, Part J: Journal of Engineering Tribology, vol. 231, no. 3, pp. 332-340, 2017.

[25] I. G. Goryacheva, Contact mechanics in tribology, vol. 61, Kluwer Academic Publishers, Dordrecht, 1998.

[26] W. Manners, "Partial contact between elastic surfaces with periodic profiles," Proceedings of the Royal Society, vol. 454, no. 1980, pp. 3203-3221, 1998.

[27] O. G. Chekina and L. M. Keer, "A new approach to calculation of contact characteristics," Journal of Tribology, vol. 121, no. 1, pp. 20-27, 1999.

[28] H. M. Stanley and T. Kato, "An fft-based method for rough surface contact," Journal of Tribology, vol. 119, no. 3, pp. 481-485, 1997.

[29] F. M. Borodich and B. A. Galanov, "Self-similar problems of elastic contact for non-convex punches," Journal of the Mechanics and Physics of Solids, vol. 50, no. 11, pp. 2441-2461, 2002.

[30] M. Ciavarella, G. Demelio, and C. Murolo, "A numerical algorithm for the solution of two-dimensional rough contact problems," Journal of Strain Analysis for Engineering Design, vol. 40, no. 5, pp. 463-476, 2005.

[31] M. Paggi and J. Reinoso, "A variational approach with embedded roughness for adhesive contact problems," 2018, https:// arxiv.org/abs/1805.07207.

[32] J. H. Tripp, J. Van Kuilenburg, G. E. Morales-Espejel, and P. M. Lugt, "Frequency response functions and rough surface stress analysis," Tribology Transactions, vol. 46, no. 3, pp. 376-382, 2003.

[33] C. Paulin, F. Ville, P. Sainsot, S. Coulon, and T. Lubrecht, "Effect of rough surfaces on rolling contact fatigue theoretical and experimental analysis," Tribology and Interface Engineering Series, vol. 43, pp. 611-617, 2004.

[34] R. L. Jackson and I. Green, "On the modeling of elastic contact between rough surfaces," Tribology Transactions, vol. 54, no. 2, pp. 300-314, 2011.
[35] Y. Xu, R. L. Jackson, and D. B. Marghitu, "Statistical model of nearly complete elastic rough surface contact," International Journal of Solids and Structures, vol. 51, no. 5, pp. 1075-1088, 2014.

[36] M. Ciavarella, G. Murolo, G. Demelio, and J. R. Barber, "Elastic contact stiffness and contact resistance for the Weierstrass profile," Journal of the Mechanics and Physics of Solids, vol. 52, no. 6, pp. 1247-1265, 2004.

[37] R. L. Jackson, "An Analytical solution to an archard-type fractal rough surface contact model," Tribology Transactions, vol. 53, no. 4, pp. 543-553, 2010.

[38] Hills D. A., Nowell D., and Sackfield A., Mechanics of elastic contacts, Butterworth-Heinemann, Oxford, UK, 1993.

[39] I. S. Gradshteyn and I. M. Ryzhik, Table of Integrals, Series, and Products, Elsevier, 8th edition, 2015.

[40] A. Arzhang, H. Derili, and M. Yousefi, "The approximate solution of a class of Fredholm integral equations with a logarithmic kernel by using Chebyshev polynomials," Global Journal of Computer Sciences, vol. 3, no. 2, pp. 37-48, 2013.

[41] J. C. Mason and D. C. Handscomb, Chebyshev Polynomials, Chapman and Hall, London, UK, 2003.

[42] K. Oldham, J. Myland, and J. Spanier, An Atlas of Functions, Springer, New York, NY, USA, 2nd edition, 2008.

[43] D. Elliott, "The evaluation and estimation of the coefficients in the Chebyshev series expansion of a function," Mathematics of Computation, vol. 18, pp. 274-284, 1964. 


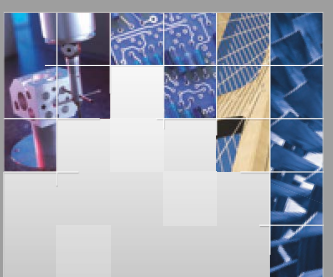

\section{Enfincering}
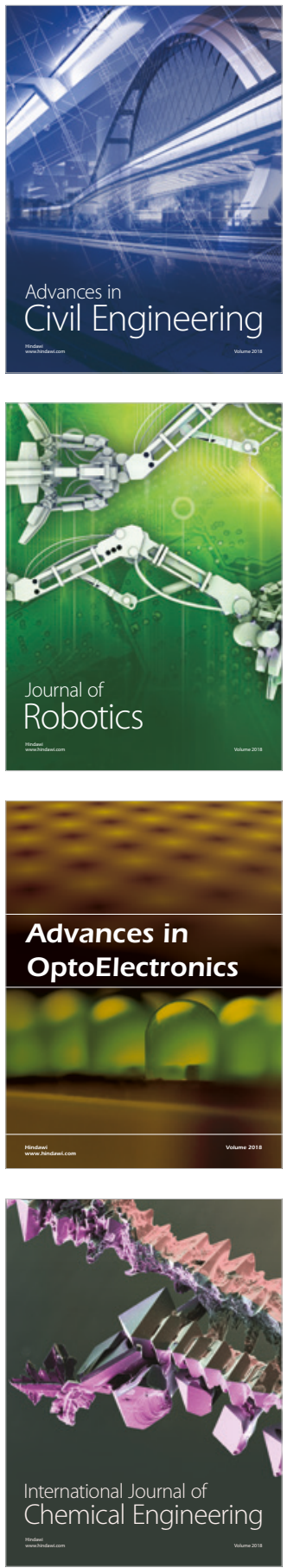

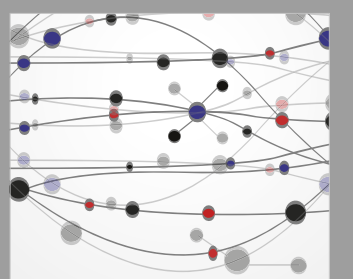

\section{Rotating \\ Machinery}

The Scientific World Journal

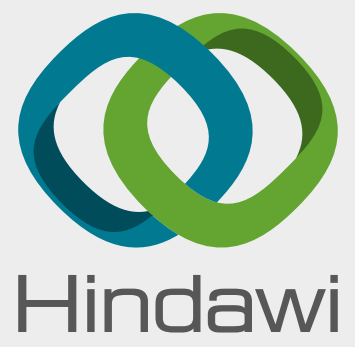

Submit your manuscripts at

www.hindawi.com
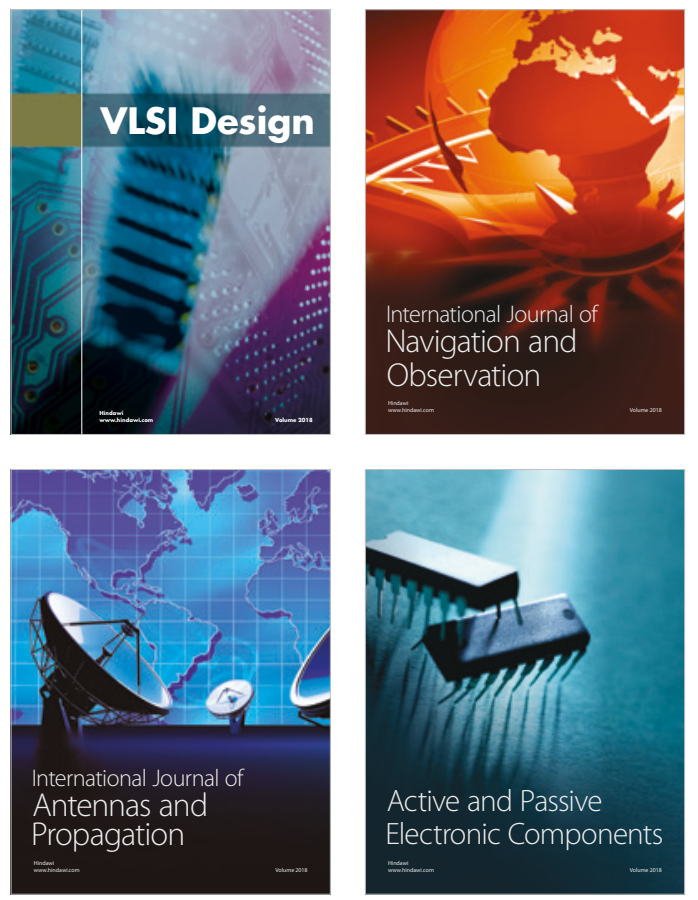
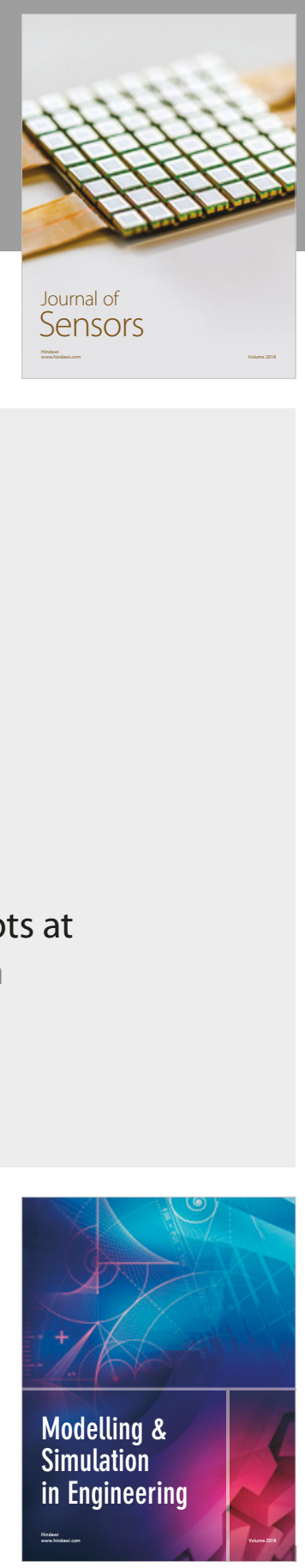

\section{Advances \\ Multimedia}
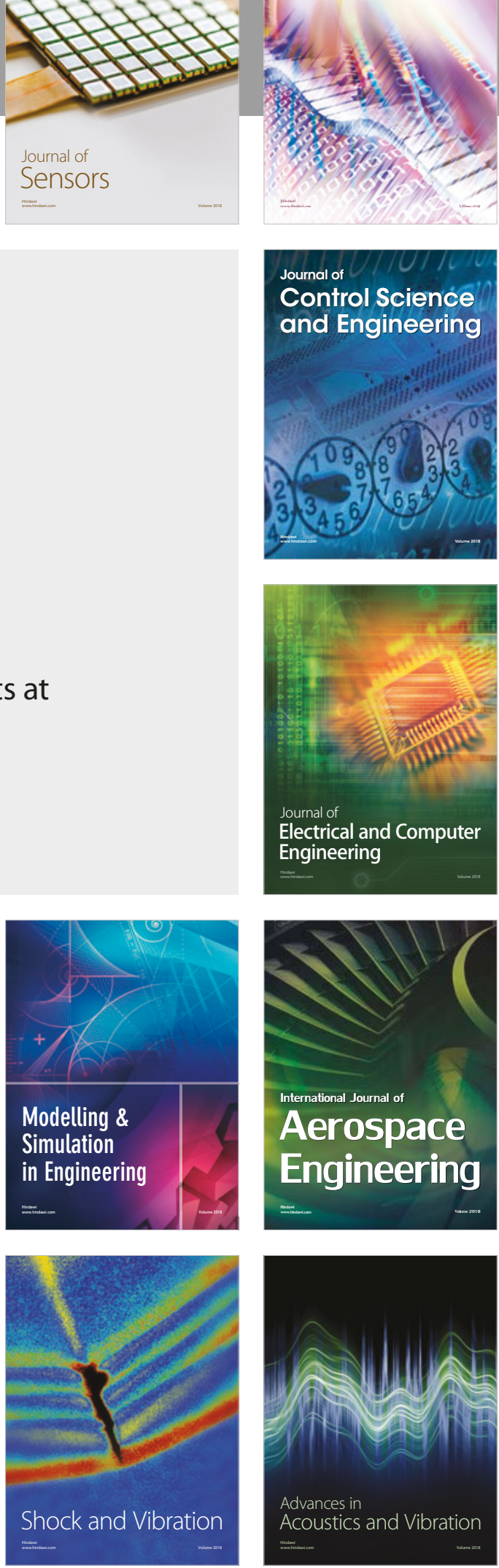